\title{
Pentax-AWS video laryngoscope for tracheal intubation in a patient with Klippel-Feil syndrome
}

\author{
Young Hyun Jo, Mi Kyeong Kim, and Keon-Sik Kim \\ Department of Anesthesiology and Pain Medicine, Kyung Hee University Medical Center, Seoul, Korea
}

Klippel-Feil syndrome (KFS) is a congenital anomaly of the cervical vertebral segments presenting three characteristics: an extremely short neck, low hairline, and significantly restricted range of motion of the neck. KFS is one of the congenital causes of difficult airway management because of these characteristic features, and patients with KFS have a risk of significant neurologic injury with minor trauma from laryngoscopy, intubation, or even positioning [1].

We hereby report a case of successful orotracheal intubation in a patient with KFS using the Pentax Airway Scope (AWS, AWS-S100 ${ }^{\circledR}$, Pentax Corporation, Tokyo, Japan).

A 34-year-old patient with KFS complicated by mental retardation and speech and hearing impairment (height, $158 \mathrm{~cm}$; weight, $58 \mathrm{~kg}$ ) was scheduled for anterior cervical discectomy and fusion of cervical vertebrae 3-4 (C3-4) and C5-6 vertebrae under general anesthesia. Physical examination revealed a short neck, low posterior hairline and restricted neck movement with minimal extension at the cervical vertebrae. The preoperative airway examination demonstrated a mouth opening of $3.5 \mathrm{~cm}$ and a thyromental distance of $4.5 \mathrm{~cm}$. Mallampati classification was grade III. Cervical spine magnetic resonance imaging revealed incomplete block, ankylosis of facet joints and compressive myelopathy of the $\mathrm{C} 2-6$. There were no remarkable findings in the chest radiograph and laboratory reports. When the patient arrived in the operating room, standard monitoring devices were applied. Pre-oxygenation was sufficiently carried out via facial mask before induction of anesthesia. Anesthesia was induced with a bolus dose of propofol $120 \mathrm{mg}$ intravenously. After confirming loss of consciousness and adequate mask ventilation, succinylcholine $75 \mathrm{mg}$ was administered for relaxation. Tracheal intubation was attempted orally with the use of the Pentax AirWay Scope. Before insertion, a well-lubricated tracheal tube was attached to a channel on the right side of a disposable transparent blade (ITL-S ${ }^{\circledR}$, Pentax Corporation, Tokyo, Japan) of the AWS. The AWS was easily inserted into the mouth with its tip toward the epiglottis and then full glottic exposure (Cormack and Lehane grade 1) was displayed on the videoscreen of the AWS (Fig. 1A). With no extension of the neck, the tracheal tube was passed through the vocal cords and smoothly advanced into the trachea (Fig. 1B). After double check of successful intubation with bilateral auscultation of breath sounds, rocuronium $40 \mathrm{mg}$ was administered intravenously for surgical relaxation. The lungs were mechanically ventilated to maintain the endtidal $\mathrm{CO}_{2}$ of 35-40 $\mathrm{mmHg}$, and anesthesia was maintained with sevoflurane in a mixture of oxygen and air at a $1: 1$ ratio. The duration of tracheal intubation with the use of the AWS was less than 20 seconds.

Awake fiberoptic bronchoscope (FOB)-guided intubation is known as the safest technique for tracheal intubation in patients with a diagnosed difficult airway [2]. Johnson et al. [3] stated that tracheal intubation with fiberoptic bronchoscopy had a high failure rate in the first attempt (approximately 53.3\%), which means it requires great skills and a lot of experience. Also, awake fiberoptic bronchoscopy requires the patient's cooperation, but the patient in this case had significant difficulty in cooperating because of the mental retardation and hearing impairment. Thus, we decided to try tracheal intubation with the AWS after induction of anesthesia using intravenous propofol. In our insti-

Corresponding author: Keon-Sik Kim, M.D., Department of Anesthesiology and Pain Medicine, Kyung Hee University Medical Center, Hoegidong, Dongdaemun-gu, Seoul 130-702, Korea. Tel: 82-2-958-8591, Fax: 82-2-958-8580, E-mail: keonsik@hanafos.com

(c) This is an open-access article distributed under the terms of the Creative Commons Attribution Non-Commercial License (http:// creativecommons.org/licenses/by-nc/3.0/), which permits unrestricted non-commercial use, distribution, and reproduction in any medium, provided the original work is properly cited. 

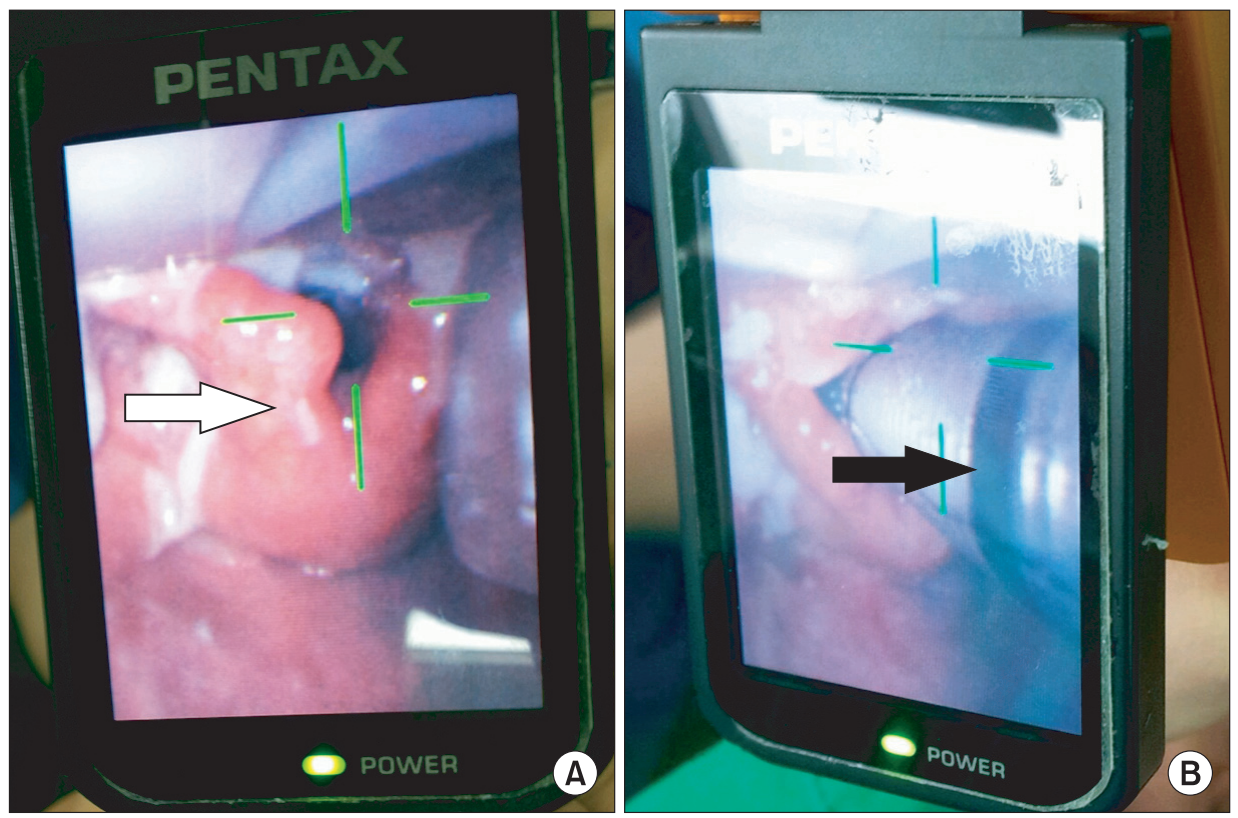

Fig. 1. (A) The target signal in the PentaxAWS ${ }^{\circledR}$ monitor was aligned with the glottic opening. The glottis was clearly and easy found. (B) The Pentax-AWS ${ }^{B}$ monitor display image of the endotracheal tube passing through the vocal cords (White arrow: Lt arytenoid, black arrow: endotracheal tube).

tution, the AWS has been frequently used in cases of predicted or unpredicted difficult airway as an alternative to fiberoptic bronchoscopy. The AWS video display unit, in combination with a disposable blade (ITL- $S^{\circledR}$ ), is a tracheal intubation device which allows indirect visualization of the vocal cords without the need to achieve a direct line of sight with the conventional "sniffing" position and leftward and anterior displacement of the tongue the hyoid bone, respectively. The intubation procedure with the AWS is monitored on a built-in liquid crystal display monitor. The ITL- $S^{\circledR}$ is anatomically designed to conform to the shape of the mouth and pharynx and is also designed to be passed over the dorsum of the tongue. Asai et al. [4] studied the effectiveness of the AWS on patients with difficult airways due to several different anatomical or pathological changes, such as restricted neck movements, deformity of the airway, and tumor in the airway demonstrating that intubation with the AWS was successful in 268 of the total 270 patients (99.3\%) with difficult Macintosh laryngoscopy. Suzuki et al. [5] reported that in an experiment with a test group of 320 people, the percentage of glottic opening score was significantly improved ( $0 \%$ to $88 \%)$ when using the AWS compared to Macintosh laryngoscopy. In most cases, a fiberoptic bronchoscope is commonly used when tracheal intubation is expected to be difficult. However, fiberoptic bronchoscopy in emergency situations can be difficult because it requires a lot of skill to perform and involves a long set-up time, and consequently entails high failure rates [2]. On the other hand, the AWS is easily handled with just brief training compared to the fiberoptic bronchoscope. However, there are some limitations to the use of the AWS. First, even with a clear view of the glottis, the tip of the endotracheal tube could become stuck at the level of the arytenoids or the epiglottis, and resultantly cannot be advanced any further. In this case, a tracheal tube can be advanced over the introducer, which can be pre-advanced through the vocal cords via the tracheal tube. Second, there are some cases when the tip of the ITL- $S^{\circledR}$ cannot be advanced towards the glottis or the posterior surface of the epiglottis. In this case, a tube can be inserted into the trachea over the introducer has been passed through the trachea via the tube, and then the tube can be separated from the ITL- ${ }^{\circledR}$. Third, the interincisor distance should be at least $2.5 \mathrm{~cm}$ because the blade of the Pentax-AWS is $1.8 \mathrm{~cm}$ thick. Lastly, the AWS cannot be used in children [4].

In conclusion, we suggest that tracheal intubation using the AWS may be a safe and fast alternative method to awake FOB intubation in patients with KFS who have a significantly high risk of a difficult airway and cervical cord injury.

\section{References}

1. Naguib M, Farag H, Ibrahim A el-W. Anaesthetic considerations in Klippel-Feil syndrome. Can Anaesth Soc J 1986; 33: 66-70.

2. Huitink JM, Buitelaar DR, Schutte PF. Awake fibrecapnic intubation: a novel technique for intubation in head and neck cancer patients with a difficult airway. Anaesthesia 2006; 61: 449-52. 
3. Johnson DM, From AM, Smith RB, From RP, Maktabi MA. Endoscopic study of mechanisms of failure of endotracheal tube advancement into the trachea during awake fiberoptic orotracheal intubation. Anesthesiology 2005; 102: 910-4.

4. Asai T, Liu EH, Matsumoto S, Hirabayashi Y, Seo N, Suzuki A, et al. Use of the Pentax-AWS in 293 patients with difficult airway. Anesthesiology 2009; 110: 898-904.

5. Suzuki A, Toyama Y, Katsumi N, Kunisawa T, Sasaki R, Hirota K, et al. The Pentax-AWS((R)) rigid indirect video laryngoscope: clinical assessment of performance in 320 cases. Anaesthesia 2008; 63: 641-7. 